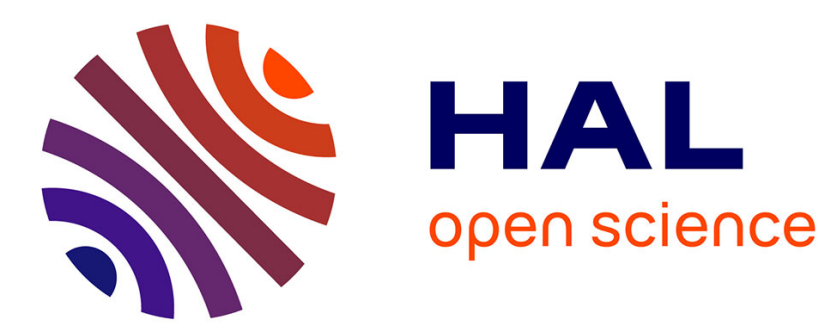

\title{
What is the reason for the asymmetry between the twins in the twin paradox? \\ Gerrit Coddens
}

\section{To cite this version:}

Gerrit Coddens. What is the reason for the asymmetry between the twins in the twin paradox?. 2019. hal-02263337v5

\section{HAL Id: hal-02263337 \\ https://hal.science/hal-02263337v5}

Preprint submitted on 3 Feb 2020

HAL is a multi-disciplinary open access archive for the deposit and dissemination of scientific research documents, whether they are published or not. The documents may come from teaching and research institutions in France or abroad, or from public or private research centers.
L'archive ouverte pluridisciplinaire HAL, est destinée au dépôt et à la diffusion de documents scientifiques de niveau recherche, publiés ou non, émanant des établissements d'enseignement et de recherche français ou étrangers, des laboratoires publics ou privés. 


\title{
What is the reason for the asymmetry between the twins in the twin paradox?
}

\section{Gerrit Coddens}

Laboratoire des Solides Irradiés, Université de Paris-Saclay, CEA-DRF-IRAMIS, CNRS UMR 7642, Ecole Polytechnique, 28, Route de Saclay, F-91128-Palaiseau CEDEX, France

23rd September 2019, updated 1st February 2020

\begin{abstract}
The true difficulty of the twin paradox does not reside in the algebra that shows that the traveling twin ages less than the twin who stays at home. The truly startling part of the paradox resides in the much more difficult question why the argument cannot be reversed by symmetry, because there is no such thing as a preferred reference frame, and motion ought to be relative. Can the traveling twin not claim with equal rights to have stayed at home while the other twin has made the journey? The answer to this question is not what anyone has thought. There is actually something very simple that has been overlooked. In drafting the protocol which defines the journey, we unwittingly pick a preferred reference frame, because we define the protocol with respect to a given frame, which thereby becomes special. It is this selection of a special reference frame which introduces the asymmetry. Hence, the reference frame wherein we define the protocol for the journey will act like an absolute frame and it is its unavoidable introduction which breaks the symmetry between the twins. There is an infinity of protocols that can be selected to define a journey and each of these journeys leads to its own corresponding twin paradox, with its own outcome as to which twin will age less. Whereas the individual trips of the two twins within a given protocol are asymmetrical, the set of all possible journeys is symmetrical, such that the symmetry of the Lorentz group is indeed respected.
\end{abstract}

PACS. $03.30 .+\mathrm{p}$ Special relativity

\section{Introduction}

The twin paradox [1] can be assumed to be sufficiently popularized to be even well known to the layman audience. There are literally hundreds of scientific papers about it. In the references section we will not present even a minute selection of them, because we will explain how in all these papers something very simple has been systematically overlooked, viz. the crucial rôle played by the definition of the journey. A nice historical overview with many references was given by Benguigui [2]. Let us call our twins Sarah and Théo. Sarah will be the twin who travels from $P$ to $Q$ and then backwards from $Q$ to $P$ at a uniform velocity $v$. Her brother Théo will be the twin who stays at home in $P$. The true difficulty of the twin paradox is not the trivial calculation which shows that according to the theory of special relativity Sarah will turn out to have aged less than her twin brother Théo. That argument only scratches the surface of the paradox. The true paradox resides in the much more difficult question why Sarah cannot claim with equal rights that she stayed at home and that it is her brother Théo who did all the travelling and therefore should be younger than her according to the very same theory of special relativity. This is a serious, fundamental question because it addresses the self-consistency of the mathematical framework. ${ }^{1}$

In many text books, the asymmetry is explained on the basis of the accelerations that inevitably must intervene at the beacons which are defining the trip. Even Einstein has claimed this. This seems to make a lot of sense. It complies with physical intuition. What else is there we could get our hands on? What else is there in the form of a telltale difference that could constitute an objective cause for the asymmetry?

Most people will feel intimidated by the turn the problem is taking this way, because there are far less physicists who are fully acquainted with general relativity than there are with special relativity. To the ones who do not master general relativity

\footnotetext{
${ }^{1}$ Many authors use space-time diagrams to argue that the twins agree on who is older. There is nothing wrong with these arguments. But they are missing the point, because they fail to lay bare the real cause of the paradox. They only confirm the asymmetry between the twins by deriving it from real cause, whose introduction is passed under silence. As already mentioned in the abstract and very clearly summarized in the second paragraph of Section 5, the real cause is the introduction of a third reference frame wherein we define the protocol for the journey.
} 
this will be felt as a curse. The task - of getting fluent in general relavity first - looks so overwhelming and time-consuming that they may feel like abandoning their desire of further inquiring into the paradox all together. For those people we can break some good news: in a well-defined setting the accelerations are irrelevant. Our arguments will be based on a rigorous mathematical inquiry rather than on misguided "physical intuition".

A very short summary of some parts of this work has been published in a Letter [3]. Here we explain in more detail how we reached the conclusions expressed in [3]. We also want to stress that the original problem is the asymmetry of the time dilatation and that it is in an attempt to popularize time dilatation that the twins and the accelerations have been introduced. These accelerations are not part of the original problem which can be formulated entirely within the context of special relativity. In fact, this introduction of the accelerations replaces the original problem by another one. The new problem is then solved in terms of these accelerations which are not part of the original problem. Therefore, while the solutions presented for the new problem may be valuable and correct within their modified context, they completely miss the point of the original problem in its pristine context. They must therefore be considered as a smoke screen that diverts the attention away from the original problem and hides the fact that solving the original problem has been eluded. As discussed in the Appendix, the discussion of the accelerations presents some problems in its own right.

\section{Kinematics and dynamics: two possible paradoxes corresponding to two possible contexts}

The mathematical framework starts from Minkowski space time $\mathbb{R}^{4}$. We consider the homogeneous Lorentz group SO(3,1), which is a set of homogeneous linear transformations of $\mathbb{R}^{4}$ which preserve the quadratic form $x^{2}+y^{2}+z^{2}-c^{2} t^{2}$. Because this quadratic form corresponds to a pseudo-metric we can call these linear transformations isometries. What we have left out here from the complete set of isometries are space-time reflections and reversals. We can extend the group $\mathrm{SO}(3,1)$ to the Poincaré group $\mathscr{L} \subset F\left(\mathbb{R}^{4}, \mathbb{R}^{4}\right)$ by adding space-time translations. ${ }^{2}$ We can then consider $\mathbb{R}^{4}$ and its Poincaré group $\mathscr{L}$ as derived from a minimal set $\mathscr{S}_{1}$ of axioms $A_{j}, j \in[1, n] \cap \mathbb{N}$, where $n \in \mathbb{N}$. The word minimal implies here that we consider the axioms as independent. For our purposes, it is not necessary to specify these axioms any further. The theory $T_{1}$, which is the set of theorems we can derive rigorously from these axioms is by construction purely mathematical. It contains only the vector space $\mathbb{R}^{4}$ and the Poincaré group $\mathscr{L} \subset F\left(\mathbb{R}^{4}, \mathbb{R}^{4}\right)$ operating on it. We may think that this is physics because it is a part of special relativity, but the reason for this is only that the theory $T_{1}$ has been proved to be the perfect mathematical model to describe those physics.

As we will see in Section 3, one can derive a twin paradox $\mathscr{P}_{1} \in T_{1}$ from the set of axioms $\mathscr{S}_{1}$. Now if we can derive a paradox from a minimal set of axioms $A_{j}, j \in[1, n] \cap \mathbb{N}$, and the axioms are not mutually contradictory, then the origin of the paradox must lie hidden somewhere inside the steps of its derivation within $T_{1}$. Solving the paradox can thus only come from an exacting scrutiny of these steps. Consequently, there must exist a solution for the twin paradox $\mathscr{P}_{1} \in T_{1}$ within $T_{1}$. This solution will not invoke accelerations to explain the paradox $\mathscr{P}_{1} \in T_{1}$, because $T_{1}$ does not contain accelerations. This stresses that there must exist a much simpler explanation of $\mathscr{P}_{1} \in T_{1}$ that is based purely on $T_{1}$.

Now for sure, accelerations can occur in an extended theory $T_{2}$. To introduce these accelerations, we need a minimal set of additional axioms $A_{n+j}, j \in[1, m] \cap \mathbb{N}$, where $m \in \mathbb{N}$. Again, for our purposes, it is not necessary to specify these axioms any further. The full set $T_{2}$ of theorems we derive from the set $\mathscr{S}_{2}$ of axioms $A_{j}, j \in[1, n+m] \cap \mathbb{N}$, and which rely on accelerations will be a part of general relativity. We have then $\mathscr{S}_{1} \subset \mathscr{S}_{2}$ and $T_{1} \subset T_{2}{ }^{3}$ Within the set $T_{2}$ there exists a second twin paradox $\mathscr{P}_{2} \in T_{2}$ wherein we allow now also for accelerations during the journeys. It is very important to realize that $\mathscr{P}_{2} \neq \mathscr{P}_{1}$, because $\mathscr{P}_{2} \in T_{2} \backslash T_{1}$ and $\mathscr{P}_{1} \in T_{1}$. We may find a solution for the twin paradox $\mathscr{P}_{2} \in T_{2}$ in $T_{2}$, but this cannot be a valid solution for $\mathscr{P}_{1} \in T_{1}$. Any explanation involving contents from $T_{2} \backslash T_{1}$ is a logically wrong explanation for $\mathscr{P}_{1} \in T_{1}$.

We will show in Section 3 how we can formulate the paradox $\mathscr{P}_{1}$ without invoking accelerations, by removing them carefully from the derivation of the paradox. As proposed above, $T_{1}$ can be considered as pure mathematics. Hence, all physical objects must be removed from the description. We must treat the problem as a "purely geometrical" one. The geometry in question is just Minkowki space-time with its Poincaré group $\mathscr{L}$ as we outlined above. All other issues (like accelerations and forces) are exterior to this "geometry" and can only be add-ons of $T_{2}$. The paradox $\mathscr{P}_{1} \in T_{1}$ addresses then a purely mathematical issue, viz. that of the self-consistency of this geometrical framework which becomes the stage for relativistic kinetics and dynamics just like Euclidean geometry and the Galileo transformations are the stage for Newtonian kinetics and dynamics. The self-consistency implies that one cannot derive a contradiction from the set $\mathscr{S}_{1}$ of axioms $A_{j}, j \in[1, n] \cap \mathbb{N}$ of the theory $T_{1}$.

People have presented this self-consistency problem in terms of twins to render the presentation more punchy and vivid. However, this way they have also introduced the accelerations because physical objects cannot change speeds without accelerations. After introducing these accelerations, the resulting problem is then no longer equivalent to the initial, purely mathematical problem of the self-consistency of the space-time geometry, because we have now introduced also elements of physics (viz. forces, masses, inertia and accelerations).

\footnotetext{
2 We use the notation $F(X, Y)$ to design the functions with definition domain $X$ that take their values in $Y$.

3 The implication $\mathscr{S}_{1} \subset \mathscr{S}_{2} \Rightarrow T_{1} \subset T_{2}$ is not always correct when one studies the foundations of set theory in mathematics, but this is not of our concern here. Our concern is to explain that we should not solve a problem of kinematics by invoking dynamics.
} 
There are thus two intrinsically different formulations of the twin paradox: a "geometrical" one $\left(\mathscr{P}_{1} \in T_{1}\right)$ and a physical one $\left(\mathscr{P}_{2} \in T_{2}\right)$. We think that by returning to the clinical essentials of the purely geometrical formulation, our presentation can gain a lot in logical rigor and didactical clarity. We have claimed here that special relativity is "geometry" because mathematicians have shown that it is a form of hyperbolic geometry. It is actually the velocity manifold (or SO(3,1)) which is hyperbolic. We do not really need to know or understand this and as explained above it does not really imply that special relativity would not be physics. It remains physics in the sense that nature tells us that it has chosen hyperbolic geometry instead of Euclidean geometry as the basic setting for its formulation. We only use the opposition geometry/physics to make it easy to discern that we should not solve a paradox by introducing concepts that that are alien to its derivation. We can equally appreciate in more familiar terms that the twin paradox can be formulated within the context of special relativity and that introducing accelerations changes the framework from special to general relativity, i.e. from kinematics to dynamics, whereby we end up with a different type of twin paradox. Although we will not address the paradox $\mathscr{P}_{2} \in T_{2}$ in our paper, we will nevertheless formulate two caveats about the arguments based on accelerations in the Appendix.

\section{Formulation of the paradox $\mathscr{P}_{1}$ without making any reference to accelerations}

In our approach we will severely limit the use we make of the full homogeneous Lorentz group $\mathrm{SO}(3,1)$, which is non-abelian and contains the group $\mathrm{SO}(3)$ of the rotations of $\mathbb{R}^{3}$. We will only use the abelian subgroup $\mathrm{SO}(1,1) \subset F\left(\mathbb{R}^{2}, \mathbb{R}^{2}\right)$ of all boosts along the $x$-axis. This implies that we only consider linear paths and ban curved paths and rotations from the considerations all together. All this is motivated by our concern to keep things as simple as possible and to avoid introducing accelerations and rotations. In fact, we will need the extension of this group $\operatorname{SO}(1,1)$ to a Poincaré-type group $\mathscr{G} \subset F\left(\mathbb{R}^{2}, \mathbb{R}^{2}\right)$ that allows also for translations in space (along the $x$-axis) and in time.

Let us consider a trip from $P$ to $Q$ at a velocity $\mathbf{v}=v \mathbf{e}_{x}$, followed by a trip from $Q$ to $P$ at a velocity $-\mathbf{v}=-v \mathbf{e}_{x}$. Of course, following standard physical thinking, there must be some short-time accelerations at $P$ and $Q$. For the rest of the time, the motion could then be uniform. But we can try to think out of the box. We will consider for this purpose a Lorentz frame $F_{1}$ that does never accelerate and always travels at speed $\mathbf{v}$ with respect to Théo. Sarah's travel is considered to start when the origin of this frame $F_{1}$ passes in front of Théo who is situated at $P$. There is one single frame $F_{1}$ of interest to us within the whole set of frames that are always travelling with uniform velocity $\mathbf{v}$ with respect to Théo in the Poincaré-type extension of $\mathrm{SO}(1,1)$. The only difference between this frame $F_{1}$ and the other frames is the moment at which it passes in $P$ in front of Théo. We can set the clock in $F_{1}$ equal to zero at the moment it passes Théo. Now we consider also the whole set of frames that always travel with uniform velocity $-\mathbf{v}$ with respect to Théo. We select the frame $F_{2}$ that passes in $Q$ at the very moment $F_{1}$ passes in $Q$. And at that moment we set the reading of the clock in $F_{2}$ equal to the reading of the clock in $F_{1}$. When $F_{2}$ will pass in $P$ in front of Théo, we can register its reading and this will define mathematically the total traveling time for Sarah. In this scheme, which is purely mathematical and cannot really be carried out by a physical being like Sarah, there are no accelerations. Our formulation and definition are obviously exempt of any reference to accelerations. Admittedly, in this abstraction we no longer discuss real physical trips with real physical twins, but we can still discuss the basic issues of time dilatation in special relativity. In fact, this alternative Gedanken experiment could also be carried out in principle. It would only require even more siblings. It is for the abstract problem formulated this way that we will solve the paradox. And as the formulation relies only on special relativity, its solution should only rely on special relativity. ${ }^{4}$

\section{Purely mathematical solution of the paradox $\mathscr{P}_{1}$ within $T_{1}$}

\subsection{Fundamental importance of the choice of the frame $F_{\star}$ used to define a journey and its end points}

The frames we have introduced are in one-to-one correspondence with the one-dimensional Poincaré-like group $\mathscr{G}$. The set of all possible frames $F_{\lambda}$ models this group $\mathscr{G}$. Each frame $F_{\lambda}$ corresponds to one transformation $L_{\lambda} \in \mathscr{G}$ of the group $\mathscr{G}$ and vice versa. If we consider this set of frames in an otherwise empty space, then it is inconceivable that there would exist some asymmetry between them because the group $\mathscr{G}$ is symmetrical. That is why uniform motion is relative. We can express these

\footnotetext{
4 The time dilatation exists already as a purely "geometrical" effect within special relativity. What we have done in searching for our approach is finding out under which conditions we can formulate the problem under its pure mathematical form, such that it reveals the intrinsic truth within the mathematical theory beyond discussion. The discussion without the accelerations is so to say an idealized mathematical theory. It takes the form of a Gedanken experiment from which we have banned any pollution of the purely mathematical argument by what we consider as physical limitations of the experimental set-up for the measurement of the ideal result. We relegate the accelerations to the status of imperfections in the design of the experimental set-up. Classical theories are always idealized in the sense that the imperfections of the measuring device do not enter the theory. This way of presenting things is of course a purely mental view. But it permits to exclude the accelerations from the idealized theory by considering them as imperfections of our experimental set-up. The set-up must be designed such as to make the relative influence of the imperfections as small as possible (as outlined in Subsection 2 of the Appendix ).
} 
intuitive notions mathematically in terms of the group elements as follows. "Absolute rest" can be attributed to a frame $F_{\star}$ by mapping it onto the identity element $\mathbb{1}$ of the group: $L_{\star} \rightarrow \mathbb{1}$. Thereby we transform all ellements $L \in \mathscr{G}$ of the group $\mathscr{G}$ by a group automorphism $\mathscr{A} \in F(\mathscr{G}, \mathscr{G}): L \in \mathscr{G} \rightarrow \mathscr{A}(L)=L L_{\star}^{-1}$. The automorphism preserves the parameters which define a relative motion, because $\mathscr{A}\left(L_{1}\right)\left[\mathscr{A}\left(L_{2}\right)\right]^{-1}=L_{1} L_{\star}^{-1}\left(L_{2} L_{\star}^{-1}\right)^{-1}=L_{1} L_{2}^{-1}$. Applying such an automorphism is actually what every observer does by claiming that, from his point of view, his frame is at rest. He will attribute then his own values to the velocities $v$ and $\gamma$-factors $\gamma=\left(1-v^{2} / c^{2}\right)^{-1 / 2}$ of other frames. The velocity $v$ and the $\gamma$-factor of the relative motion between any pair of such frames will be preserved by the automorphism.

Therefore, as it is not the reference frames which can break the symmetry inherent to relative motion, the only thing left to produce the paradox is the definition of the protocol for the journey. This journey is defined with respect to some chosen reference frame $F_{\star}$. It is the choice of this protocol frame as an "absolute frame" that breaks the symmetry between a pair of frames. We can appreciate how this happens as follows. We consider frames $F_{1}$ and $F_{1}^{(\lambda)}$ that are passing in $P$ and in $P_{\lambda}$ when the time $t$ on Théo's clock is zero. In the whole set of frames $F_{1}^{(\lambda)} \in \mathscr{G}$ with velocity $\mathbf{v}$ the distance $P Q$ is Lorentz contracted, which explains why Sarah ages less. This is due to the fact that the whole of Sarah's trip is defined by end points $P$ and $Q$ in Théo's frame. Théo's frame $F_{T}$ functions thus as the absolute protocol frame $F_{\star}$. It is in this frame that the journey will start putting the clocks to zero, that the end points points $P$ and $Q$ of Sarah's trip are defined, and that the clocks will be compared in the end. The whole protocol is defined in his frame. By identifying Théo's frame $F_{T}$ with $F_{\star}$, we break the symmetry between the twins. As a matter of fact, the end points $P$ and $Q$ of Sarah's trip are at rest in Théo's frame $F_{T}=F_{\star}$. The whole trip is defined in Théo's frame. It is thus really defining the trip by beacons in Théo's frame which introduces the asymmetry. This frame $F_{\star}$ is of course not really absolute, An observer moving with respect to it can equally consider that his frame $F_{O}$ ought to be "absolute". But we can make all calculations with respect to the frame $F_{\star}$ and translate them to the frame $F_{O}$ by an automorphism. And this will treat correctly all relative motion and preserve the answer to the question who ages less, such that we can calculate everything with respect to $F_{\star}$.

Now to reverse the argument to make it symmetrically valid for Sarah, we must define the trip differently. We must define the trip by beacons in space-time that are not defined in Théo's frame but in Sarah's frame. They should be at rest in Sarah's frame. ${ }^{5}$ For the moment, we just point out that there are no physical objects available to us that could play the rôle of the beacons required to define the trips. All the physical objects we have at our disposal and that could play the rôle of beacons belong to Théo's frame. To define the "symmetrical trip" of Théo with respect to Sarah, we must therefore introduce an unbiased set of purely mathematical beacons that extends the set of physically real objects that we have at our disposal to define trips, because this physical set is biased.

The beacons correspond also to traveling frames and group elements. We would have to launch rockets from remote positions in space at well chosen times to obtain objects that could serve as physical objects embodying the beacons which Sarah may need to define Théo's symmetrical trip. As this is not very practical, we consider the beacons as mathematical objects. With the appropiate mathematically defined beacons, it will be Théo who ends up as the younger twin when the trips over.

\subsection{The true solution of the paradox}

In the following, we will allow both twins to travel simultaneously with respect to a protocol frame. To avoid confusion, we will use the term "trip" to design the journey of an individual twin, and "journey" to design the set of both trips.

[1] Special case. Let us point out that we can even define the end points of the history in a frame $F_{\star}$ that travels at such a speed that Théo seems to travel at a speed $-\mathbf{w}$ and Sarah at a speed $\mathbf{w}$ with respect to it. Following Galilean logic this frame would have velocity $w=v / 2$, but relativistically the value of $w$ is different, due the addition rule for velocities in the Lorentz group, such that $w$ is rather defined by the second-degree equation $v=2 w /\left(1+w^{2} / c^{2}\right)$. This equation applies both to the to and fro parts of the relative motion. The equation has two algebraic solutions $w=\frac{c^{2}}{v} \frac{\gamma \pm 1}{\gamma}$, where $\gamma=\left(1-v^{2} / c^{2}\right)^{-1 / 2}$ as usual. The solution with the plus sign must be discarded because it would lead to superluminal velocities $w \geq c$. One can easily convince oneself that the other solution always implies $w \leq c$. If we defined the journey appropriately with beacons at rest in a frame $F_{\star}$ with this velocity $w$, then Théo and Sarah would have to travel in opposite directions at a speed $w$ with respect to this frame $F_{\star}$ and they would end up with the same age. ${ }^{6}$

[2] General case. To make an in-depth study of the asymmetry (see Fig. 1), we first choose a reference frame $F_{\star}$ wherein the two beacons we will use to define the trips are at rest. All calculations can be made by formulating the trips with respect

\footnotetext{
${ }^{5}$ At first sight this may look like a bemusing statement due to the fact that Sarah's trip corresponds to two frames and Théo has only one frame within the formulation as it stands now. But we will explain in Subsection 4.2 how we can remove this confusion in a more general formulation based on Fig. 1. In this more general formulation, the identity $F_{T}=F_{\star}$ that ties $F_{T}$ to $F_{\star}$ will be lifted.

${ }^{6}$ It is better to specify the speed of the relative motion always in the protocol frame $F_{\star}$, because the turning points of the two twins, which are simultaneous in the protocol frame $F_{\star}$, are no longer simultaneous in the reference frames of the twins.
} 

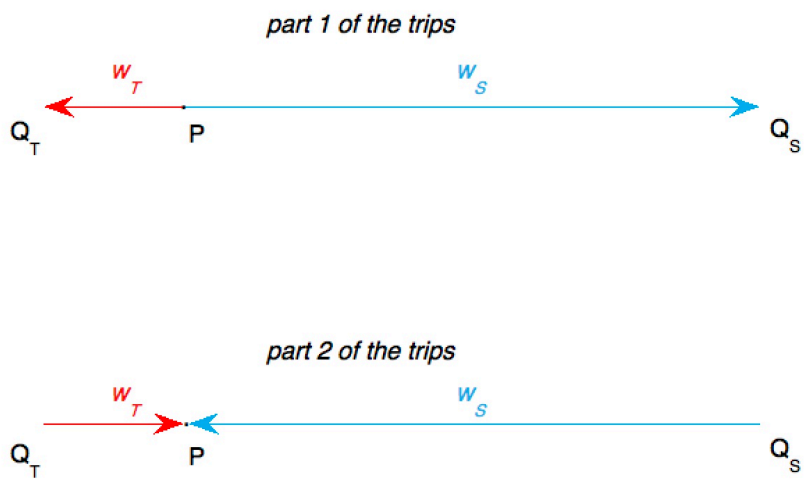

Fig. 1. The generalized scheme for the trips of Théo and Sarah in the reference frame wherein we define the protocol. In this protocol frame the beacons at $Q_{T}$ and $Q_{S}$ are fixed. In the first part, the twins move out. In the second part they join one another again. Both parts of the journey have the same duration within the protocol frame $F_{\star}$. The classical twin paradox is obtained in the limit $w_{T} \rightarrow 0$. The point $Q_{T}$ coincides then with $P$. The symmetrical journey, where Théo ages less, is obtained in the limit $w_{S} \rightarrow 0$. $Q_{S}$ coincides then with $P$. But in this symmetrical journey Théo's relative motion with respect to Sarah is not the same as in the journey that corresponds to the limit $w_{T} \rightarrow 0$ (See Section 5). We can consider a whole continuum whereby the relative motion occurs with velocity $v=\left(w_{S}+w_{T}\right) /\left(1+w_{S} w_{T} / c^{2}\right)$, and the ratio $w_{T} / w_{S} \in\left[0, \infty\left[\right.\right.$. One can even extend this to $\left.w_{T} / w_{S} \in\right]-\infty, \infty[$, if we allow the twins to move out in the same direction.

to this reference frame $F_{\star}$. They can be translated by Lorentz transformations to any other frames. But we will not present any of these boring calculations because they are straightforward and well-known. Towards this frame Sarah can have a speed $w_{S}$ and Theo a speed $w_{T}$, while (with the proviso given in Footnote 6) the relative speed of Sarah to Theo is $v=$ $\left(w_{S}+w_{T}\right) /\left(1+w_{S} w_{T} / c^{2}\right)$. All time dilatations can be calculated from the relative velocities with respect to the protocol frame $F_{\star}$ and are of some form $t^{\prime}=t / \gamma$, where $\gamma$ can take the values $\gamma_{T}$ or $\gamma_{S}$. We adjust the lengths of the trips of Sarah and Theo in such a way that their turning points are simultaneous within the protocol frame. Having two absolute values for the relative velocities within the protocol frame is actually not forbidden, but we want to keep things as simple as possible. This way the two twins will travel the same amount of time in the protocol frame. In the more specific cases $w_{T}=0$ and $w_{T}=w_{S}$ mentioned above these constraints on the lengths are implicit, because one length is zero, or both lengths are equal. In the traditional twin paradox we are thus identifying the protocol frame $F_{\star}$ with Théo's frame $F_{T}$. In the generalized formulation this ambiguity is lifted. With hindsight we can appreciate that the identification is the culprit of the paradox. The protocol frame $F_{\star}$ is "absolute" (logical proposition $p\left(F_{\star}\right)$ ), while Théo's frame $F_{T}$ is not (logical proposition $\neg p\left(F_{T}\right)$ ). It is identifying the two frames $\left(F_{\star} \equiv F_{T} \equiv F\right)$, which transforms $p\left(F_{\star}\right) \& \neg p\left(F_{T}\right)$ into the puzzling contradiction $p(F) \& \neg p(F)$ of the paradox.

We can also define the journeys in the reverse way, by imposing $v$ and a ratio $w_{S} / w_{T}$ like we did in the special case where $w_{S}=w_{T}=w$, by calculating to which frame $F_{\star}$ corresponds from these parameters (but we must formulate here the same proviso as in Footnote 6). After having fixed this problem we can then have $w_{S}>w_{T}$ such that Sarah ages less, $w_{S}=w_{T}$ such that both twins age at the same rate, or $w_{S}<w_{T}$ such Théo ages less. Each of these cases entails a different twin paradox. This will make it much more obvious that we are bound to pick a biased set of beacons to define a journey, and that this surreptitiously introduces a priviliged frame $F_{\star}$ that seems to define some absolute rest. It is to the definition of the journey, not to any of the twins, that we convey here a status of absoluteness. Of course, as explained above - by considering an observer with a frame $F_{O}$ - this is only an allowed convention, a subjective viewpoint or a perceptional illusion. The bias we have fallen prey to in the standard presentation of the twin paradox is due to the contents of the set of physical objects (with their inertia) that are available to us to define the protocol and its frame. With the mathematical beacons we can define journeys at will. ${ }^{7}$ For each paradox one can eliminate the accelerations by replacing both Sarah and Theo by two frames whose origins pass the beacons at the right times in the protocol frame. In the limits $w_{S} \rightarrow 0$ or $w_{T} \rightarrow 0$ one of the sets of two frames corresponding to one of the twins coalesces into a single frame. However, mathematically, we should continue to count them as two frames. We can compare this to what we tell about a point where a straight line is tangent to a circle. The two intersection points coalesce here also into a single tangent point. For the sake of generality, this single point must

7 One could even define a journey by two beacons that are not at rest in a same frame, but we will not consider this complication. 
mathematically also be considered as two (admittedly coalescing) points. In such a general formulation, the protocol we use to define the trips of the two twins becomes then also general, with each twin always requiring two frames. Physically, this is not farfetched, because in reality Théo's velocity $w_{T}$ may never be identically zero but just negligibly small. We should allow him to keep on living normally and move around on Earth while Sarah is on her relativistic two-way trip to Alpha Centauri. Our generalization shows that asymmetry between the twins can certainly not be explained by refering to the number of frames one needs to describe the trips. In the protocol frame there are always two frames of relative motion, such that from this point of view the situation is truly symmetrical. The description in the protocol frame $F_{\star}$ can be translated to any other Lorentz frame $F_{O}$. We can define an infinity of journey protocols, leading to an infinity of twin paradoxes. There is thus not just one twin paradox, but an uncountable infinity of them.

\subsection{In cauda venenum}

We can dissect the paradox even further. It is obvious that the to and fro parts of his trip contribute equally to the ageing of a twin. The twin paradox can be split into two parts (explicitly visualized in Fig. 1) for which the algebra intervening in the calculation of the time dilatation is identical. Both halves just embody a paradox $\mathscr{P}_{0}$ of the time dilatation over a distance $P Q$. The twin paradox is thus equivalent to the juxtaposition of two algebraically identical time dilatation paradoxes $\mathscr{P}_{0} \in T_{1}$. And the time dilatation paradoxes $\mathscr{P}_{0}$ on the one-way trips $P Q$ boil down to exactly the very same question as for the twin paradox: Why should Sarah not be entitled to state that it is Théo who must be subject to time dilatation, because motion is relative? Now the two halves of the twin paradox can both be formulated without invoking any accelerations, just by considering frames that are permanently in uniform motion as we did in Section 3 and as is done in text books. The paradox $\mathscr{P}_{0}$ can thus be formulated in $T_{1}$ without requiring any mathematical constructions on our behalf as was the case for $\mathscr{P}_{1}$ in Section 3. It can therefore be entirely solved within $T_{1}$. But despite the fact that $\mathscr{P}_{0}$ clearly belongs to $T_{1}$, we have ended up discussing an illustration $\mathscr{P}_{2}$ of it within $T_{2}$. Solving $\mathscr{P}_{0}$ in $T_{2}$ is actually logically flawed, because it should not involve elements of $T_{2} \backslash T_{1}$. Hence, in all our goodwill to render the time dilatation paradox more eloquent and fascinating to the layman by presenting it under the more enthralling guise of an eye-catching twin paradox, we have unwittingly spoiled the initial simplicity of the problem by introducing turning points. By smuggling in the accelerations, which are totally irrelevant to the initial time dilatation paradox, these turning points have subsequently become the focus of all our attention, transforming the whole well-meant communication effort ironically into a self-inflicted smoke screen.

Allowing for accelerations in the calculations is certainly possible but as we explained above, this is an independent problem in its own right. We are switching then from solving the geometrical paradoxes $\mathscr{P}_{0}$ or $\mathscr{P}_{1}$ to solving the physical paradox $\mathscr{P}_{2}$, and from special relativity to general relativity. For the initial purpose of illustrating and discussing the time dilatation paradox, this is totally scatterbrained and self-defeating. This is most eminently not the way we should explain or illustrate time dilatation to our students in an introductory course about special relativity. In fact, a large part of the solution has consisted in merely deconstructing again the whole razzle-dazzle part of the presentation, just to enable ripping apart the smoke screen it has generated. What has been introduced largely for the sake of popularizing time dilatation for the masses has turned out to add only unnessary further algebraic and conceptual complexity. And alas, this distraction of added complexity has functioned as dust in the eyes, concealing the real solution of the paradox $\mathscr{P}_{1}$ and the fact that we have never been able to nail down this solution. It has been a red herring that made us all look in the wrong direction.

\section{The difference between relativistic and Galilean symmetry of relative motion}

We finally want to point out that some confusion can be created by our classical intuition based on Newtonian mechanics, where the relative motions conceptually just take the mathematical expressions $\mathbf{r}(t)$ (for Sarah's motion with respect to Théo) and $-\mathbf{r}(t)$ (for Théo's motion with respect to Sarah). We may embark that intuition unconsciuosly with us at the back of our mind in our quest for understanding. We must raise here a red flag! Because in relativity this is plain wrong, as the two twins have different schemes of simultaneity and length. For the problem at hand, we must rule out any such subliminal appeal to our classical intuition right from the start by a conscious act of rejection. The symmetry between the journeys the two twins are carrying out is not of the Newtonian or Galilean type. The symmetry that prevails in relativity is of a different type. In relativity, the set $S$ of all possible journeys is symmetrical just like in Galilean logic such that it contains both $\mathbf{r}(t)$ and $-\mathbf{r}(t)$. But in relativity the symmetry does not follow the Galilean logic all the way, because for a given journey, $-\mathbf{r}(t)$ does no longer represent the motion of Théo with respect to Sarah if the motion of Sarah with respect to Théo is $\mathbf{r}(t)$. It is rather of a type $\mathbf{r}^{\prime}\left(t^{\prime}\right)$, where $\mathbf{r}^{\prime}$ and $t^{\prime}$ must be carefully detailed, and certainly is not equal to $-\mathbf{r}(t)$. In other words, the expected relativistic symmetry duly exists but it applies to the complete set $S$ of possible journeys, while the detailed Galilean symmetry $\mathbf{r}(t) \leftrightarrow-\mathbf{r}(t)$ between the trips of the two travelers in a single journey does no longer prevail. Very obviously, we should never have doubted about all this because $\mathrm{SO}(1,1)$ and $\mathrm{SO}(3,1)$ are groups and therefore automatically symmetrical, because all groups have a corresponding automorphism group. After introducing a prefered frame $F_{\star}$, the group $\mathscr{G}$ will still remain symmetrical with respect to this frame. As explained above, choosing a prefered frame is just like identifying its group element $L_{\star}$ with the identity element by mapping it on this identity element by a group automorphism $\mathscr{A}: L \rightarrow L L_{\star}^{-1}$. 
Important note. Many authors use space-time diagrams to show why both Sarah and Théo must agree who is older. While this shows that the two trips in a single journey are in general indeed asymmetrical, this is not the solution of the paradox we are treating in this paper. As we said in the introduction this "solution" only scratches the surface. It merely renders the algebraic calculations we called trivial in the introduction more anschaulich by representing them under a more visual pictural form. It just translates the algebra into geometry and shows that the calculations are self-consistent, such that we can obtain the exact result in several non-conflicting ways. While this proof of the self-consistence of the calculations is correct, it would be a cheat to sell this proof as the solution of the true twin paradox. The true paradox we wanted to solve is why this de facto asymmetry can be compatible with the general notion that all motion must be relative. We can observe that this "solution" only confirms the asymmetry that has already beforehand been laid down in the protocol, because the space-time diagram tacitly rests on the asymmetry introduced by the protocol of the journey. The origin of the true paradox is that we identify two types of symmetry: the symmetry between the two individual trips within a journey and which is broken by the definition of that journey, and the symmetry that corresponds to the notion that motion should be relative. The latter corresponds to the symmetry of the set of all possible journeys. In Fig. 1 we run through this set by moving $P$ between $Q_{T}$ and $Q_{S}$, and the set is symmetrical with respect to the centre $C$ of $Q_{T} Q_{S}$. On the other hand, the position of $P$ on $Q_{T} Q_{S}$, and therefore the definition of a particular journey, will only be symmetrical provided $P=C$. The confusion that leads to the paradox stems from the fact that the identification between the two types of symmetry mentioned is exact in Galilean kinematics while wrong in relativistic kinematics.

\section{Conclusion and final remarks}

In conclusion, this is the first logically exact and spot-on solution of the twin paradox. Surpisingly, the asymmetry is just produced by the definition of the journey. In trying to define such a journey we have just been fooled by a bias that exists within the set of the physical objects available to us in the physical world that surrounds us and which could serve as possible beacons for trips. But the correct full set of all possible beacons must be unbiased and very obviously reflect the full symmetry of the Lorentz group. The lesson we must learn from this is that we should be suspicious of "physical intuition" when we are trying to solve paradoxes, because - most of the time - paradoxes resort to purely logical issues. What we should rely on is therefore rather cold, robotic logic than leaping to conclusions based on human (or physical) intuition.

A less polished version of [3] was submitted on the 9th of August 2019 to the American Journal of Physics, because I thought that its audience of physics teachers might discry some grace and pedagogical merrits in the elegant simplicity of the solution discovered. But beauty is in the eye of the beholder, as the editor, Richard H. Price, rejected the paper out of hand justifying his decision by stating: "You seem to be denying the fact that time dilation is physically real. It has, in fact, been confirmed by many experiments". Abhishek Agarwal (Physical Review Letters) denied me any possibility of recourse by bluntly stating without any further explanation: "Your manuscript has been considered. We regret to inform you that we have concluded that it is not suitable for publication in any APS journal". Similar disempowering tactics were used by Jessica Thorn (European Journal of Physics). These are of course gems to be preserved for future generations as a testimony about decisionmaking based on spreadsheets and key performance indicators of excellence extracted from ORCID data base surveillance.

\section{Appendix: Two possible logical errors we can make in trying to explain $\mathscr{P}_{2}$}

\subsection{Boobytrap 1: Sarah does not forcedly feel her accelerations}

In this subsection we want to show that an argument often used in the discussion is wrong. Many approaches are based on the narrative which has it that Sarah must accelerate her spacecraft at the points $P$ and $Q$, that she can bring about the accelerations needed by firing her rockets, that she will then feel these accelerations and that this in turn will clearly show that the accelerations could be responsible for the asymmetry. And when we fail to imagine any other paradigm to solve the paradox, we readily make the leap that they should be responsible for it. Well, they are not! The whole narrative is a leading argument and not in the least compelling. Firing the rockets of a spacecraft is not the only way we can accelerate it. To illustrate this we can refer to the way NASA reduces the cost of bringing satellites to distant planets, by putting to profit accelerations exerted by other planets along a carefully planned trajectory. These accelerations are gravitational and as such not necessarily felt. A spacecraft in circular orbit around the Earth is in an accelerated motion and the people inside do not feel anything of this motion: They are "weightless". This is so symmetrical that in the analogous problem of the motion of the Earth around the Sun we have believed for millenia that the Earth was standing still with the rest of the Universe revolving around us. If we replaced the Sun by a black hole and we kept far enough away from it in order not to feel tidal effects at the length scale of our bodies, we could circle around that black hole at relativistic velocities and think we are standing still because we do not feel any acceleration: In the language of general relativity we would be travelling on a geodesic. The argument contains thus a pars pro toto. One cannot always notify to Sarah that she should admit that she has felt accelerations in order to convince her that she would not be entitled to turn the tables by claiming that from her viewpoint it was Théo who made the travel. Of course, 
felt or otherwise, all accelerations will introduce additional effects of time dilatation in addition to those of special relativity. Our considerations about their contributions must intervene in the analysis of the paradox $\mathscr{P}_{2} \in T_{2}$, but it is absolutely not our intention to address the solution of $\mathscr{P}_{2} \in T_{2}$. We suspect that the accelerations may displace the problem rather than solving it, because under certain conditions accelerated motion can also be relative, as illustrated by the examples given.

\section{Boobytrap 2: The time dilatation during accelerated motion has no bearing on the time dilatation during uniform motion}

The following argument shows how we can render the relative contribution of the accelerations to the age difference negligible in a physical experiment with real twins. Let us consider a straight line segment $P Q$ as the trajectory of the journey. We can make the accelerations take place over short distances $P P_{1}, Q_{1} Q, Q Q_{1}$ and $P_{1} P$. The motions over $P_{1} Q_{1}$ and $Q_{1} P_{1}$ are then uniform. Whatever the effect of the accelerations may be, we can choose the distance $P_{1} Q_{1}$ and make it so long that the effect of the accelerations becomes negligible with respect to the age difference that builds up during the uniform motions over $P_{1} Q_{1}$ and $Q_{1} P_{1}$. This is is because the age difference builds up linearly along the distance $P_{1} Q_{1}$. Due to the homogeneity of space, increasing the distance $P_{1} Q_{1}$ will not change the effect of the accelerations over $P P_{1}, Q_{1} Q, Q Q_{1}$ and $P_{1} P$. We can thus make the age difference as large as we like by increasing the distance $P_{1} Q_{1}$, a fact that must condemn any quantitative attempt to account for the assymetry between the two twins on the sole basis of the accelerations. The time dilatations due to the accelerations and those due to the uniform motion are completely independent. Each part of the journey must be considered as making a contribution in its own right, if we want to end up with a viable account of the global time dilatation. This shows that attributing the asymmetry uniquely to the accelerations is a five-star glaring error and raises the question of the true origin of the asymmetry when the motion is strictly uniform, as is the case for time dilatation within the pure context of special relativity.

\section{References}

1. E.F. Taylor and J.A. Wheeler, in Spacetime Physics, Freeman, New York (1963).

2. L. Benguigui, "The tale of two twins", arXiv:1212.4414 (2012) and references therein.

3. G. Coddens, Eur. Phys. J. Plus 135:152 (2020). 